\title{
OLDER WOMEN'S VIEWS ON THEIR PARTICIPATION IN A BLENDED EDUCATIONAL PROGRAM USING FB ROOM PLATFORM
}

\author{
Venetis Kanakaris ${ }^{1}$, \\ Pavlis-Korres Maria ${ }^{2 \mathrm{i}}$ \\ ${ }^{1}$ Postdoc Student, \\ Aristotle University of Thessaloniki, \\ Greece \\ ${ }^{2}$ Associate Professor, SEDEd, \\ Aristotle University of Thessaloniki, \\ Greece
}

\begin{abstract}
:
Several studies have found that elderly people have a difficulty in using social media platforms and smartphone apps due to a number of barriers that discourage them from engaging with technology. This study presents older women's views regarding a twomonth blended (synchronous and asynchronous) learning course on using apps and social media on smartphones in their daily life. The design of the educational course was based on older adult women's needs. The findings of the qualitative research indicate that older people's educational needs are better met when they participate in an asynchronous e-learning environment, where they are given the opportunity to proceed at their own pace. Moreover, the results suggest that designers and educators can incorporate video tutorials (narration and subtitles) in the learning programs as an effective educational mean and use Facebook as a learning platform so as to engage learners and facilitate communication between educators and learners.
\end{abstract}

Keywords: e-learning, Facebook room, video-lessons, blended learning, smartphones

\section{Introduction}

The development of technology and the subsequent advent of smartphones can potentially improve the quality of older people's lives in a number of ways (Carpenter \& Buday, 2007; Chen \& Persson, 2002; White et al. 2002). An expanding number of studies suggest that the use of social media and internet training may enhance older adults' psychosocial well-being (Cotten, Ford, Ford, \& Hale, 2012, 2014; Shapira, Barak, \& Gal, 2007; White et al. 1999).

i Correspondence: email vkanakar@nured.auth.gr, mariapavliskorres@nured.auth.gr 
Although social media use and internet browsing have been shown to have a potentially positive impact on the psychology of older adults, it seems that they are still reluctant to adopt the technology. With the development of touchscreen technology in smartphones and tablets - which older people might find easier to work with - various useful and handy applications (apps) are now running on smartphones. Given how older adults are likely to want more frequent communication with their children via social network apps and how younger adults are keen on using them (Zhou, Rau, \& Salvendy, 2014), it is vitally important to deal with the perspectives, impacts, and learning needs of older adults, who only have limited internet experience and skills.

Even though previous research findings have implied that healthcare smartphone apps may improve patients' health management among older adults (Boulos, Wheeler, Tavares, \& Jones, 2011; Grindrod et al. 2014), it seems that research focused on the influence of other types of apps (such as entertainment and communication apps) on older adults' physical/ psychological well-being is lacking. The majority of existing studies on older adults learning about touchscreen technology have been conducted in pure research settings, in the context of testing a highly scripted model targeted at a homogeneous group of older adults (Zhou et al. 2014). Further research focusing on the learning needs of older people on issues they tend to struggle with, like the use of touch screen technology in their daily life, is needed. The study of an apps-based touchscreen device training course can serve as an important source of information regarding how best to design and disseminate it within the gerontechnology ecosystem (WHO, 2002; Zheng et al., 2015).

In this article, the basic elements of a two-month blended (synchronous and asynchronous) educational program are presented, as well as the views of older women who took part in it, regarding its content and the way it was implemented. The aim of the program was to teach older women how to use smartphone applications that can facilitate their daily life. Among other things, participants were taught how to compose and send emails with attachments, top-up their balance on Cosmote app, as well as basic FB functions - namely group creation, website, live video and FB Room, posting and sharing to enhance communication and social networking. The program was designed based on an analysis of learners' educational needs. Preliminary research was conducted, investigating the educational needs of older people from two elderly care centers in Northern Greece intending to attend an educational program on using smartphones as a communication tool to join social media networks. Research findings indicated that elderly women were more willing to attend an educational program than men. Key areas of their interest included access to the internet (e.g., online payments), creating an email account and sending emails, and joining social media to help alleviate feelings of isolation by communicating with individuals or groups with the same interests (Kanakaris \& Pavlis Korres, 2020). The initial plan was to design face to face teaching lessons with older women, but the concept was redesigned due to the COVID-19 pandemic and, after consulting with the participants, a blended e-learning course was implemented. 


\section{Literature Review}

Mobile devices have the potential to enrich the lives of older people. Smartphones and tablets offer access to the internet and a variety of applications that can help people stay in touch with others and acquire information relevant to their needs (Gao et al., 2015; Ma, et al., 2016). According to Tsai et al. (2015), mobile devices allow older adults to feel connected to the world and their families and up to date with current digital trends, to keep up with special interests that can help them develop positive attitudes toward social life, as well as, to pursue a meaningful lifestyle during late adulthood. Although the adoption of mobile devices by older adults has increased dramatically in recent years, the majority of older people only use limited functions and have not fully understood the beneficial aspects. Adult educators are aware of the need to provide educational activities for them that will help them learn how to use mobile devices to improve their quality of life. Most importantly, incentives need to be articulated to encourage older adults to become familiar with new technologies and ensure their continued use of them. After reviewing related literature, Mackowicz and Wnek-Gozdek (2016) highlighted three main reasons that motivate older people to undertake education opportunities for learning new technology: obtaining skills useful in daily life, acquiring new knowledge, and socialization. Learning topics associated with communication tools, social media, digital photography, health, and medicine, and financial management are especially intriguing and helpful for elderly learners (Boulton-Lewis et al., 2007; Zheng, Spears, Luptak, \& Wilby, 2015; Klimova, Simonova, Poulova, Truhlarova, \& Kuca, 2016; Mackowicz \& Wnek-Gozdek, 2016; Kanakaris \& Pavlis Korres, 2020). Therefore, it is important for instructional designers to design learning materials that meet the practical needs of older adult learners using mobile devices. Miller (2016) illustrated how learning content can be helpful for seniors toward mastering portable tablet devices so as to browse the web, handle (send/receive) emails, navigate social media, organize their calendars, read e-books, magazines and newspapers, record and edit videos, navigate maps, keep healthy, manage files, online security, etc. By tapping into these recommendations, senior education institutes should design courses focusing on these issues and their applications and encourage older adult learners to use mobile devices as ubiquitous learning tools.

Moreover, another way to help seniors get acquainted with smartphone applications is to develop manuals and tutorials that focus on ameliorating the basic learnability of mobile applications designed for older adults (Digmayer \& Jakobs, 2012). Designing interactive video tutorials for the elderly that adapt to existing applications will help them learn mobile applications more independently and at their own pace (Chen et al, 2017). Digmayer and Jakobs (2012) demonstrated how many seniors, especially those without prior relevant experience, prefer video tutorials with embedded help text. Their findings also suggested that video tutorials used by elderly people should not be more than 2 minutes long, as seniors tend to forget what they have just watched and can find this material boring or tedious. 
Due to the current trend of the rapidly ageing population, countries like Taiwan, where a white paper entitled "Towards an aged society: Policies on education for older adults" was published in 2006, are stressing the lifelong learning rights of older adults and highlighting the liabilities of all sectors (public, private, non-profit) with the aim of providing learning opportunities to the elderly and subsequently contribute to the successful aging of their citizens (Hung \& Lu, 2014; Lin \& Huang, 2013; Wang, 2017). The COVID-19 pandemic limited older people's access to face-to-face teaching and ignited a rapid move to online learning. Social media platforms such as Facebook provide a wide range of advantages for students and can be used as a supplementary tool for distance learning (Chugh \& Ruhi, 2018).

The learning process in distance education works best when participants are involved in the activities of the online classroom and participate in the discussion that takes place. In addition, engaging and involving students in the various activities of the online classroom allows them to take control of their own learning (Manca \& Delfino, 2021). By remotely engaging in online discussions, learner control is facilitated, since participants can choose who they engage with (Ulla \& Perales, 2020). This means that learners become the centre of the learning process, instead of the instructor. The content of the learning can be determined by the learners who can also decide the nature of the communication. Furthermore, relevant studies emphasize that the integration of adult learning principles and the conditions for effective adult learning in an online learning environment should be a priority in the training of teachers who will be employed or are employed in adult e-learning programmes. (Pavlis Korres, Karalis, Leftheriotou, \& Garcia Barriocanal, 2009; Pavlis Korres \& Leftheriotou, 2016; Pavlis Korres \& Leftheriotou, 2020). Social media platforms such as the Facebook could be used to enhance learner engagement in the learning process. Furthermore, Facebook has been found to be a potentially functional tool in social learning, distance learning, as well as in arts education. Distance learning instructors should adopt an interest in using Facebook, but only with suitable educational material. Therefore, using Facebook as an online educational platform during the COVID-19 pandemic facilitates active learning and enhances community building, which in turn can help mitigate feelings of "isolation" in students (Greenhow \& Chapman, 2020; Ulla \& Perales, 2021).

\section{Methodology}

\subsection{The Study}

This study is a part of a post-doc research focusing on the educational needs of the elderly and, the design, implementation, and evaluation of an educational program on the use of smartphones along with the possibilities they offer in improving older adults' daily lives and promoting communication and social networking. Initially, research was conducted in order to determine the content of the program and other elements regarding its implementation (Pavlis Korres \& Leftheriotou, 2020). The research was conducted in two Elderly Care Centers (E.C.C.), sited in different cities in the region of Eastern Macedonia 
and Thrace in Greece. The Elderly Care Center provides elderly people with health care services, fitness programs, and a variety of educational programs. Initially, the research sample was comprised of 20 older people from the two E.C.C.s. and the results from this research showed that they want to learn how to use social media, web-banking, and how to send emails from their smart-phones during face-to-face lessons. After the outbreak of the Covid-19 pandemic, the participants in the research were reduced from 20 persons to 7 older women. Even though the original plan was to provide face-to-face lessons to the elderly, things changed after the unfortunate passing of two women who contracted the virus. As a result, after consulting with participants, the researcher decided to design a new blended educational program based on video lessons (asynchronous mode) and FBRoom (Facebook Room) (synchronous mode) and to incorporate educational material that participants could work with remotely, such as video lessons with narration via Youtube. The first step was to develop a pilot video lesson which would subsequently be evaluated be 2 women and one expert, who would provide feedback. The next step was to take the feedback into consideration and improve the video lessons. Overall, 8 videos were created, lasting between 1 minute and 15 seconds to 4 minutes and 15 seconds. The videos were created to meet the needs of the women, as determined during the needs assessment process, and their content is presented in Table 1.

Table 1: Video tutorials basic information

\begin{tabular}{|c|l|c|c|c|}
\hline & Title/Content & Duration & Subtitles & Narration \\
\hline 1 & Compose and send email & $1 \mathrm{~min} 17 \mathrm{sec}$ & $\checkmark$ & $\checkmark$ \\
\hline 2 & Compose and send email with attached file & $1 \mathrm{~min} 18 \mathrm{sec}$ & $\checkmark$ & $\checkmark$ \\
\hline 3 & $\begin{array}{l}\text { Instructions on how to navigate the Cosmote (Greek } \\
\text { Telecom Service Provider) application for mobile } \\
\text { balance top-up }\end{array}$ & $4 \mathrm{~min} 25 \mathrm{sec}$ & $\checkmark$ & $\checkmark$ \\
\hline 4 & Share a post on Facebook & $1 \mathrm{~min} 26 \mathrm{sec}$ & $\checkmark$ & $\checkmark$ \\
\hline 5 & Create a room on FB & $2 \min 19 \mathrm{sec}$ & $\checkmark$ & $\checkmark$ \\
\hline 6 & Create Live Video on Facebook & $3 \min 20 \mathrm{sec}$ & $\checkmark$ & $\checkmark$ \\
\hline 7 & $\begin{array}{l}\text { Instructions for creating a Website on FB via Android } \\
\text { SmartPhone }\end{array}$ & $2 \min 20 \mathrm{sec}$ & $\checkmark$ & $\checkmark$ \\
\hline 8 & Create a Facebook Group & $1 \min 53 \mathrm{sec}$ & $\checkmark$ & $\checkmark$ \\
\hline
\end{tabular}

A blended (synchronous/asynchronous) teaching method based on the Facebook platform was chosen, where a closed group together with the Facebook Room covered the communication needs between the learners and educator. The duration of the course was eight weeks and the first week featured an introduction to the video lessons and instructions on how to use Facebook (FB) Room. The researcher prepared and provided video lessons with narration as well written instructions within the videos and the FB Room, created to promote communication between the educator and the learners.

Each week, learners were provided with a video lesson that they studied at their own pace, at a time of their choosing. In a synchronous meeting through the Facebook Room, the learners and the educator communicated in real time discussed difficulties they were faced with during video tutorials and exchanged views and experiences. 


\subsection{Participants}

The participants were 7 old women, aged between 61 and 74. Table 2 included the demographic profiles of the participants regarding age range and educational level.

Table 2: Age range and educational level of the participants in the course

\begin{tabular}{|l|c|c|c|}
\hline \multirow{2}{*}{ Educational Level } & \multicolumn{3}{|c|}{ Number of Women/Age range } \\
\cline { 2 - 4 } & $\mathbf{6 1 - 6 5}$ & $\mathbf{6 6 - 7 0}$ & $\mathbf{7 1 - 7 4}$ \\
\hline Elementary School & 2 & 1 & 1 \\
\hline High School & & 1 & 1 \\
\hline Technical College & & & \\
\hline University & 1 & & \\
\hline
\end{tabular}

Due to its reduced size, the sample is not fully representative of elderly people, and thus no generalizations can be made, but it is interesting, in that it is self-representative and provides necessary and useful information for the design of a blended educational course that is being addressed to the elderly.

\subsection{Research Methodology}

The methodology that was chosen was the qualitative research approach (Cohen, Manion, \& Morrison, 2013), which means that fundamental data was gathered using interviews as a research method. The interviews were conducted by the researcher via telephone and FB-Room by specific appointment with each interviewee. Each interview was semi-structured, lasted between 15 to 20 minutes and consisted of open-ended questions (Cohen, Manion, \& Morrison, 2013) The research questions concerned the participants' views on whether the educational materials and the way they were presented met their needs, their views on modern education, and whether they preferred e-learning (modern and asynchronous) to face-to-face learning.

Thematic analysis, a research method of describing and quantifying phenomena as well analyzing documents (Nowell et al., 2017; Braun \& Clarke, 2012), was used to analyze the data. The thematic analysis allows the researcher to examine theoretical issues so as to enhance understanding of the data. By using the thematic analysis, it is possible to refine words into fewer content-related categories. It is assumed that when classified into the same categories, words and phrases share the same meaning (Elo \& Kyngäs, 2008). Thematic analysis is a method that is mainly described as "a method for identifying, analyzing and reporting patterns (themes) within data" (Braun \& Clarke,2006, p. 79). The aim is to achieve a concentrated and broad description of the phenomenon, and the result of the analysis is concepts or categories that describe the phenomenon. Usually, the scope of these concepts or categories is to create a model, conceptual system, conceptual categories (Stemler, 2015). 


\section{Findings and Discussion}

For the reader's convenience, the answers to the main research questions are presented in figures/tables containing the basic codes and themes that emerged from the thematic analysis, while quotes from the interviews are also provided.

Figure 1 presents the basic themes that emerged in relation to learners' views on the positive impact of video lessons.

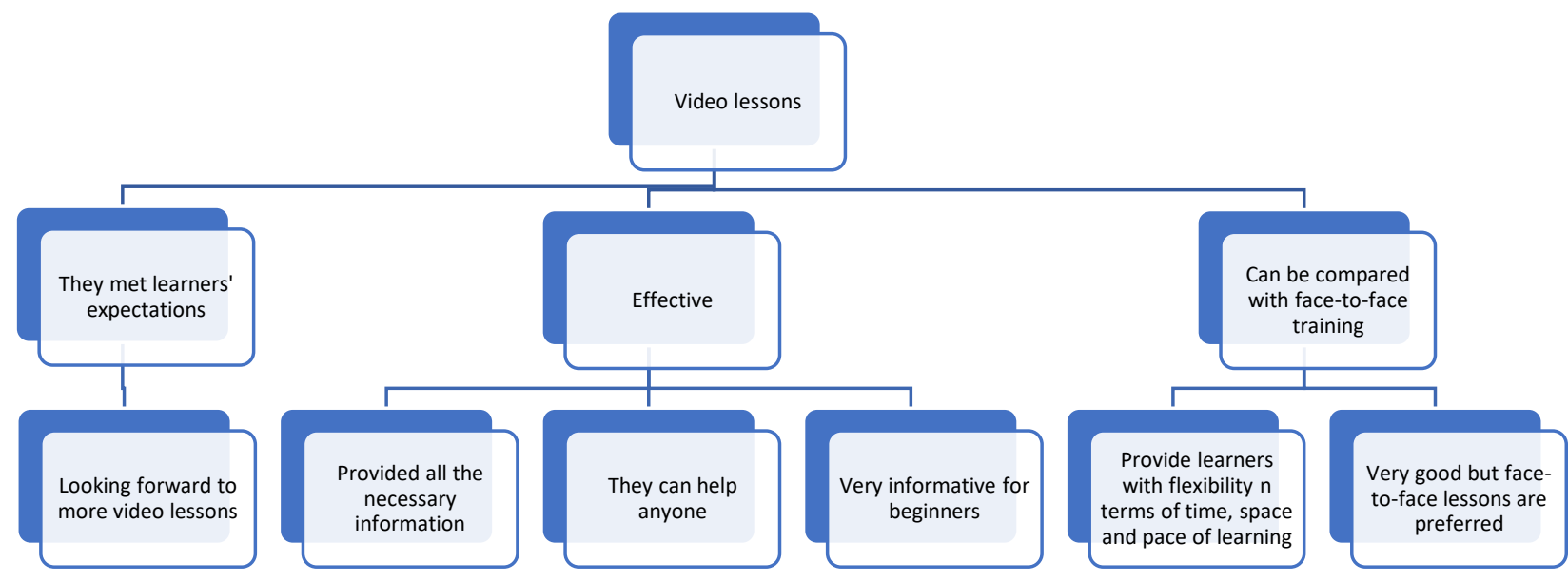

Figure 1: Learners' views on the positive impact of video lessons

The thematic analysis shows that learners recognize that the asynchronous educational mode -specifically the video tutorials- which can be accessed anywhere and everywhere via YouTube, were effective and helped them understand the fundamentals of each topic. Among other things, participants mentioned: "These videos are a great tool for someone that wants to learn the basics" (L5), "Very good work, the videos can help anyone" (L3) and "The videos are very good I expect to be provided with more video tutorials" (L7). Video lessons met participants' expectations, "This was what I was expected"(L1), whereas according to L2, they were unexpectedly informative: "I didn't expect them to be like this, the videos gave me all the information that I needed». Given that the video lessons were created based on learners' needs and suggestions, the results were similar to Miller's findings (2016), showing that seniors learn better when courses are tailored to their needs and conducted through a suitable medium. Moreover, these findings are consistent with previous studies regarding the appeal of distance learning to older adults (Dorin, $\mathrm{M}$, 2007; Githens, 2007; Mulenga \& Liang, 2008). The abovementioned studies suggest that older people's needs are better addressed by asynchronous e-learning because the latter facilitates autonomous learning and working at one's own pace. This piece of information 
can be used when designing lessons on the use of smartphones for older people. In a similar vein, the findings of this study showed that, due to the ease of access via YouTube and the embedded narration and subtitles, video lessons might prove to be useful in a blended learning programme as they help participants learn easier and faster (Digmayer \& Jakobs, 2012; Chen et al, 2017). Women's views on the effectiveness of video lessons compared to face-to-face lessons tend to be similar, "I believe that they are almost on par with face-to-face lessons"(L4), and they also seemed to appreciate the flexibility in terms of time and location that they offer, "I watched most videos twice, but the best thing was that they were all on YouTube, which meant I could watch them whenever and wherever I wanted" (L3). This suggests that a properly designed educational program can be beneficial to elderly learners.

Participants' views on the main video features and the number of views each learner required are presented in Tables 3 and 4 respectively.

Table 3: Learners' views regarding video tutorials features

\begin{tabular}{|c|c|c|c|l|}
\hline & \multicolumn{4}{|l|}{ Video tutorials features } \\
\hline Interviewee & Narration & Subtitles & Speed/Flow & \\
\hline 1 & $\uparrow$ & $\leftrightarrow$ & $\uparrow$ & "The narration and speed were great" \\
\hline 2 & $\uparrow$ & $\uparrow$ & $\uparrow$ & "The videos had no problems" \\
\hline 3 & $\leftrightarrow$ & $\leftrightarrow$ & $\uparrow$ & "The flow was excellent as well as the videos" \\
\hline 4 & $\uparrow$ & $\uparrow$ & $\leftrightarrow$ & $\begin{array}{l}\text { "The subtitles and the narration were an excellent } \\
\text { idea" }\end{array}$ \\
\hline 5 & $\leftrightarrow$ & $\leftrightarrow$ & $\uparrow$ & $\begin{array}{l}\text { "The flow was great and it allowed me to instantly } \\
\text { practice on the smartphone what I was seeing in the } \\
\text { videos" }\end{array}$ \\
\hline 6 & $\uparrow$ & $\leftrightarrow$ & $\uparrow$ & $\begin{array}{l}\text { "When I watched the videos for the first time, I } \\
\text { thought that the flow of the instructions was good, } \\
\text { but, in my opinion, the subtitles were redundant" }\end{array}$ \\
\hline 7 & $\uparrow$ & $\uparrow$ & & "The way the videos were developed was excellent" \\
\hline
\end{tabular}

$\uparrow:$ Positive aspect

$\leftrightarrow:$ Neutral aspect

$\downarrow$ : Negative aspect

Table 4: Number of views each learner required

\begin{tabular}{|c|l|c|}
\hline Number of views each learner required & $\begin{array}{c}\text { Number of } \\
\text { Interviewee }\end{array}$ & 3 \\
\hline 1 & "I had to watch most video tutorials three times to understand the steps" & 2 \\
\hline 2 & "I watched most video tutorials twice to fully comprehend them" & 2 \\
\hline 3 & $\begin{array}{l}\text { "I watched most videos twice, but the best thing was that they were all on } \\
\text { YouTube, which meant I could watch them whenever and wherever I wanted" }\end{array}$ & 3 \\
\hline 4 & "I had to watch the tutorials three times to learn all the steps" & 3 \\
\hline 5 & "I watched the video tutorials only three times" & 3 \\
\hline 6 & "I watched the tutorials three times to fully understand the process" & 3 \\
\hline 7 & "In most cases, I watched the videos three times to be able to follow the steps" & \\
\hline
\end{tabular}

The information in Tables 3 and 4 shows that the three main features based on which the tutorials were designed make them a very good learning tool, whereas participants seem 
to be able to learn the steps of the process by watching the videos up to three times on average. Learners were very pleased with the way the videos were produced and the features they offered, namely narration and subtitles, and the fact that they were on YouTube, which made them available at any time. These results are consistent with the findings of Digmayer and Jakobs (2012) and Chen et al. (2017), who concluded that videos should contain multiple pieces of information. These studies have also found that the duration of video lessons plays a very important role, as they can be tedious if they are too long. As shown in Table 1, 6 out of 8 videos that were used for this study were shorter than 2 minutes and 30 seconds, since the goal was for them to contain all the necessary information in the shortest possible time. As far as the speed of the video tutorials $s$ concerned, participants claimed they were satisfied with it, as is the case with the written instructions, which they found conductive to the flow of the video lessons. Moreover, many of the women revealed they needed no more than 3 viewings per video lesson in order to feel confident they understood it. These findings are in line with former research (Chaffin \& Harlow, 2005) supporting that older adults can acquire new abilities and skills in the same way younger ones can, but they normally learn at a slower pace and need more repetitions.

With respect to whether the video lessons met the learners' needs, women's views are presented in Figure 2.

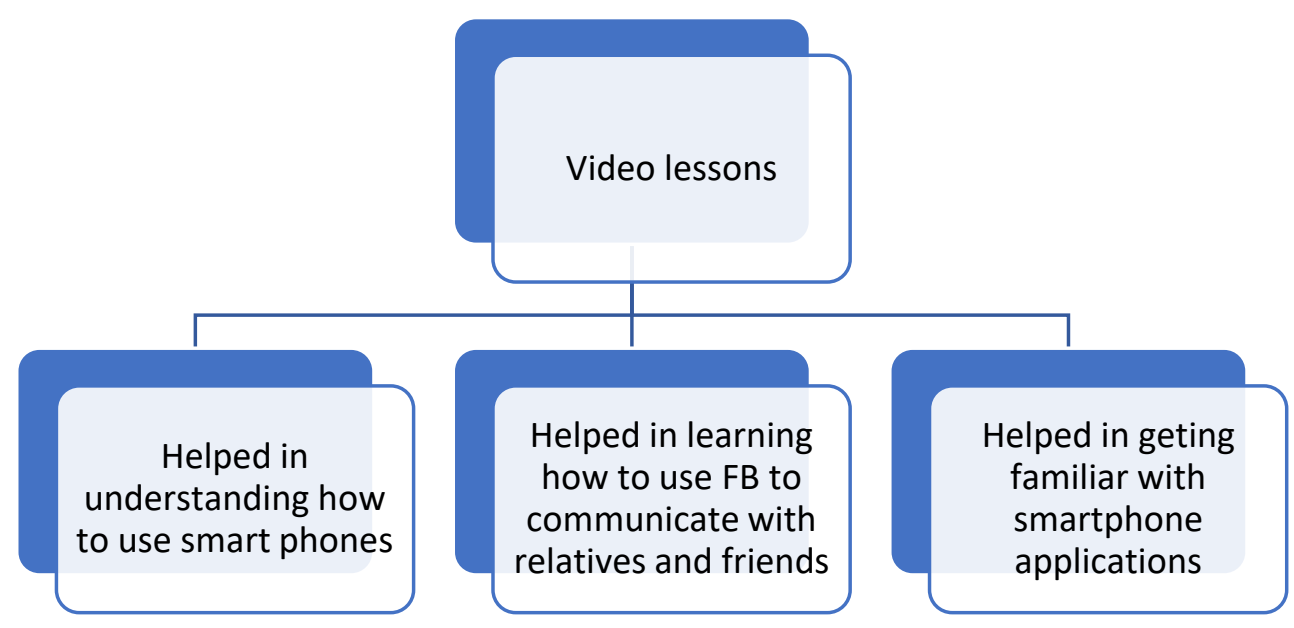

Figure 2: Video lessons response to learner needs

Figure 2 shows that the video tutorials provided all the information the learners had requested, incorporating it in the best possible way. Among other things, participants said: "The videos provided the instructions I needed" (L4), "All the videos provided the help I needed" (L5). Moreover, the thematic analysis reveals that the video tutorials assisted learners in device use and familiarized them with applications. This is also evident in their comments: "These videos were exactly what I wanted. They helped me get over my fear of using applications" (L1), "The videos taught me how to use smartphones more easily" (L2), "The videos were excellent and made me understand that the smartphone is a very useful tool" (L7). 
Additionally, not only did participants learn how to use the medium (FB Room) during the session, but some of them adopted it in their personal life in order to communicate with their loved ones. One woman in particular stated: "Thanks to these videos I can now talk to my grandson on Facebook" (L3). The above results are corroborated by those of Tsai et al. (2015) and Mackowicz and Wnek-Gozdek (2016), who suggest that older people are motivated to learn new things when they can use them in their everyday life, particularly when they learn things about maintaining or strengthening their family and social relationships through communication with relatives and friends.

Women's views on the synchronous learning mode via the Facebook Room, are illustrated in Figure 3.

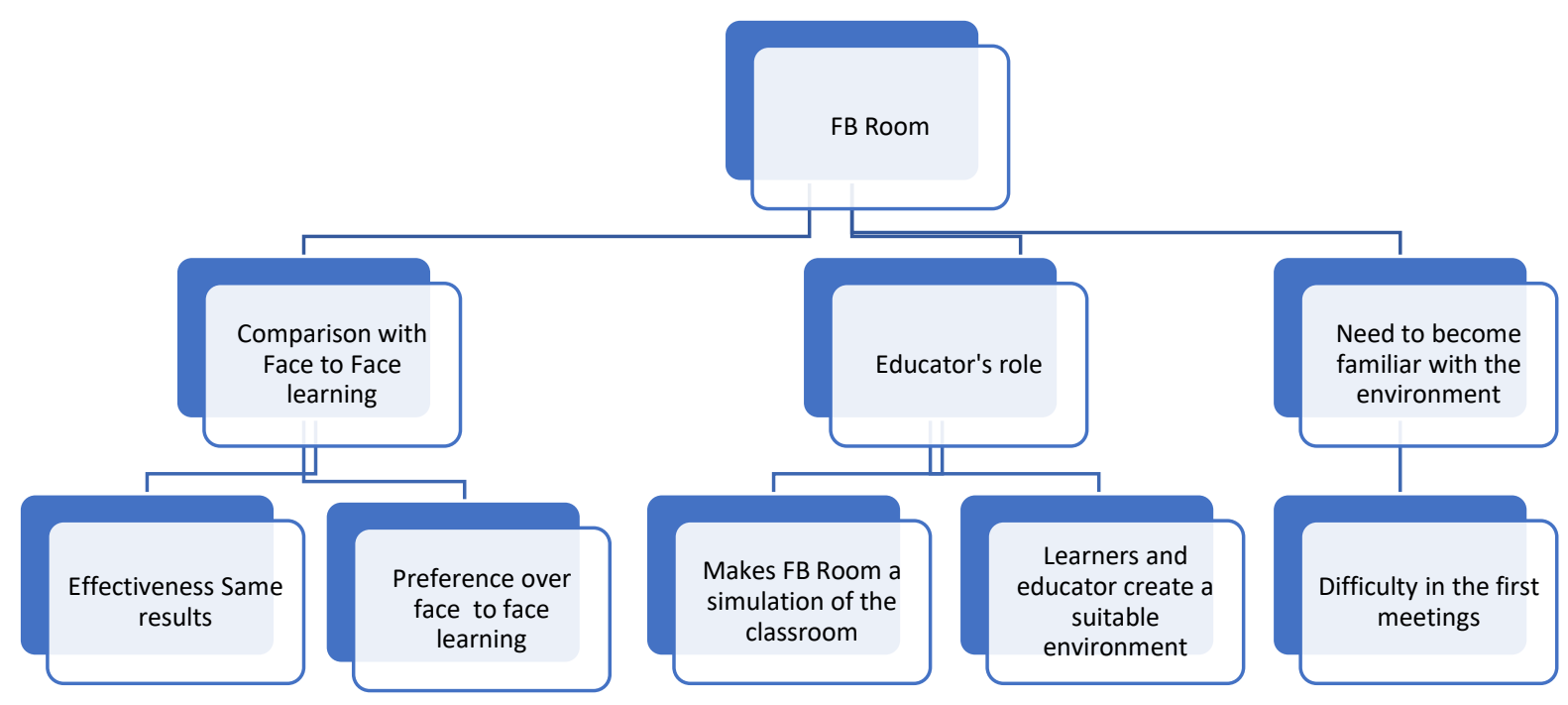

Figure 3: Online lesson on FB Room versus face-to-face lessons

As shown in Figure 3, the results form data processing revealed that, with the exception of one case, learners embraced Facebook Room as a teaching tool, and even though some of them found it difficult to navigate at first, they soon felt more comfortable and even compared distance learning during the pandemic to the traditional classroom. Four women, in particular, said: "Even though the class was moved online, we were still able to learn new things" (L2), "FB Room was a bit different from the classroom, especially at the beginning, but the goal is the same. To learn new things" (L3), "At the beginning I was not happy, but after the second online session, things got better and I got used to it" (L4), "Trying new things always seems difficult at first, but in the end, I felt distance learning is not so strenuous after all" (L5). Further, with the guidance of the instructor, learners became increasingly engaged in the learning process. According to two older women, for example: "The educator used the same teaching methods they use in the classroom, but the medium -FB room-was different" (L1), "FB room was not the same as the classroom, but the educator and the learners made it happen and we managed to continue the training program throughout the COVID-19 pandemic" (L5). These results are consistent with the findings of Greenhow and Chapman (2020) and Ulla and Perales (2021), who suggested that Facebook as an educational platform can promote active learning and community building and it can help reduce learners' feelings 
of isolation during the distance learning process. Although most of the participants found distance learning very similar to face-to-face courses, one older woman argued that distance learning did not provide the right environment compared to the traditional classroom: "Distance learning was not as comfortable as face-to-face learning. I still prefer faceto-face lessons" (L6).

The elderly women's views on the extent to which communication was promoted in Face Book Room lessons, and the factors that influenced it.

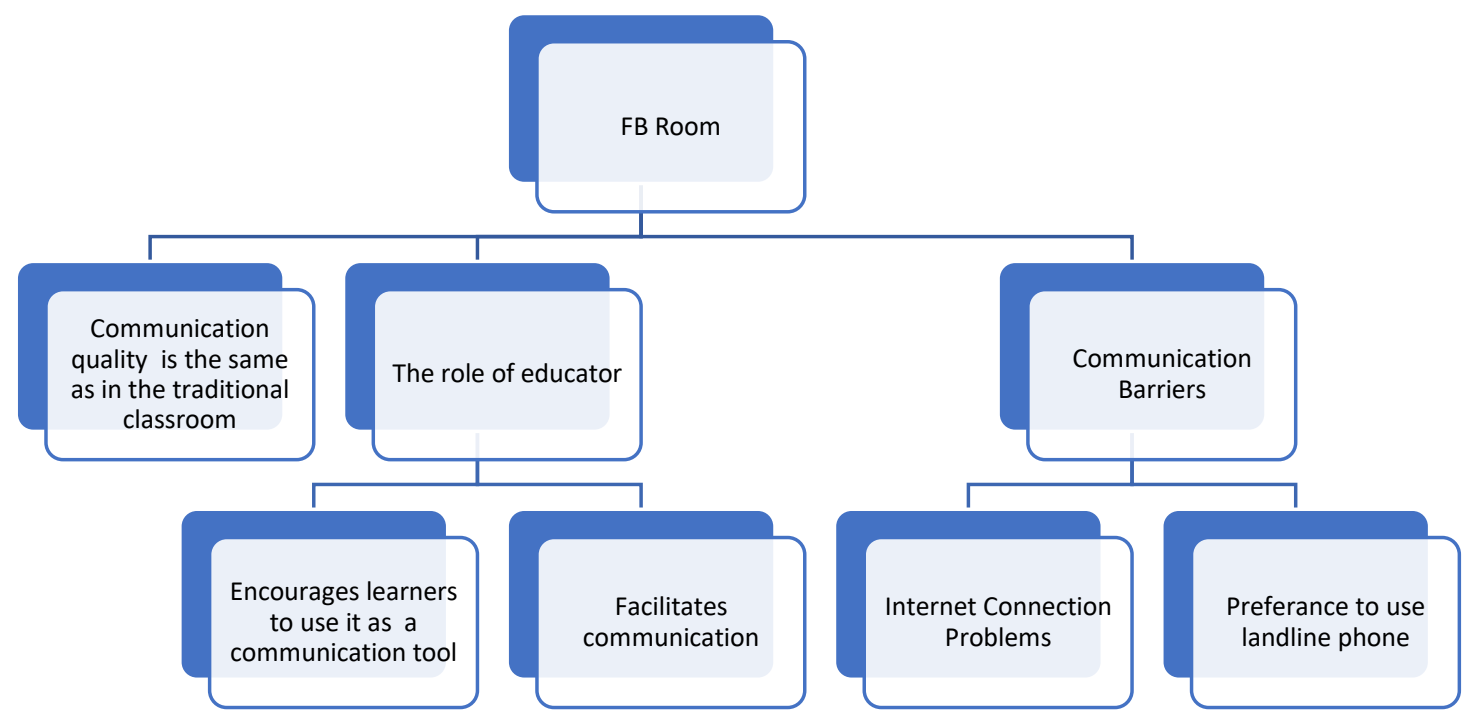

Figure 4: Older women's views about the medium (FB Room)

The processing of the collected data, as presented in figure 4, revealed that, despite the fact that FB Room was initially an unfamiliar environment for participants, the educator managed to help them engage more actively in the learning process of distance learning. The results showed that the educator played a crucial role during the learning process as their guidance and encouragement allowed learners to adapt smoothly the online environment and to engage to the learning process (Greenhow \& Chapman, 2020; Ulla \& Perales, 2021). The results are consistent with the literature on the important role of the instructor and the need for appropriate training in order for them to be able to integrate adult learning principles in an online environment and to successfully carry out the demanding role of the instructor. (Pavlis Korres, Karalis, Leftheriotou, \& Garcia Barriocanal, 2009; Pavlis Korres \& Leftheriotou, 2016, Pavlis Korres \& Leftheriotou, 2020). Döş (2007) and Swift (2018) explored the impact of online discussions on the communication between the educator and the learners. Their studies also indicated that the use of Blogs and social media promote communication and collaboration between the learners and the instructor. The majority of women learners who participated in this study (5/7) confirm this claim, highlighting the usefulness of online discussions and social media as a means of communication, and the facilitating role the instructor can play: "The communication between me and the educator was the same as it was in the classroom" (L1), "In the beginning, I was little intimidated to ask questions during the online lesson, but the educator 
encouraged me, as he always does, and urged me and the rest of the learners to try to not only use $F B$ during the lessons but incorporate it in our every-day life too, so as to communicate with many people" (L2), "Although FB Room was very new to us, the instructor helped us navigate it and use it as a communication tool" (L3), "The educator still uses the same methods and speaks to us like they do during face-to-face lessons" (L4), "Using FB Room in the learning process helped us become familiar with a new communication tool without losing contact with other learners and the educator" (L5). By the second week of the learning program, more and more learners had started to behave in the same way as during face-to-face lessons. These findings are consistent with those of Manca and Delfino (2021) and Ulla and Perales (2020), who concluded that when learners are engaged in online discussions during distance learning, they start adopting the same behaviors they had in the traditional classroom, they take control of their own learning and they are placed in the centre of the learning process, rather than the educator. Further, the results showed that some of the learners started using FB Room as a communication tool to connect with relatives and friends. One woman, in particular, said: "Our educator showed us how to use this great tool, FB Room, which makes distance learning feel like we are in the classroom, and we also found a new way to easily communicate with more than one person" (L7). Although most learners found Facebook-Room to be a very good communication tool, one of them found it hard to navigate and said they had to use the landline multiple times, in order to contact the educator: "In my opinion, FB Room was a difficult communication platform, although I learned how to partially use it by the end of the course. During the lessons, I sometimes had to use the landline to communicate with the educator" (L6).

Reaching the end of this section in order to help the reader understand better the findings of this study the themes that emerged from the data process are presented in whole in Figure 5. 


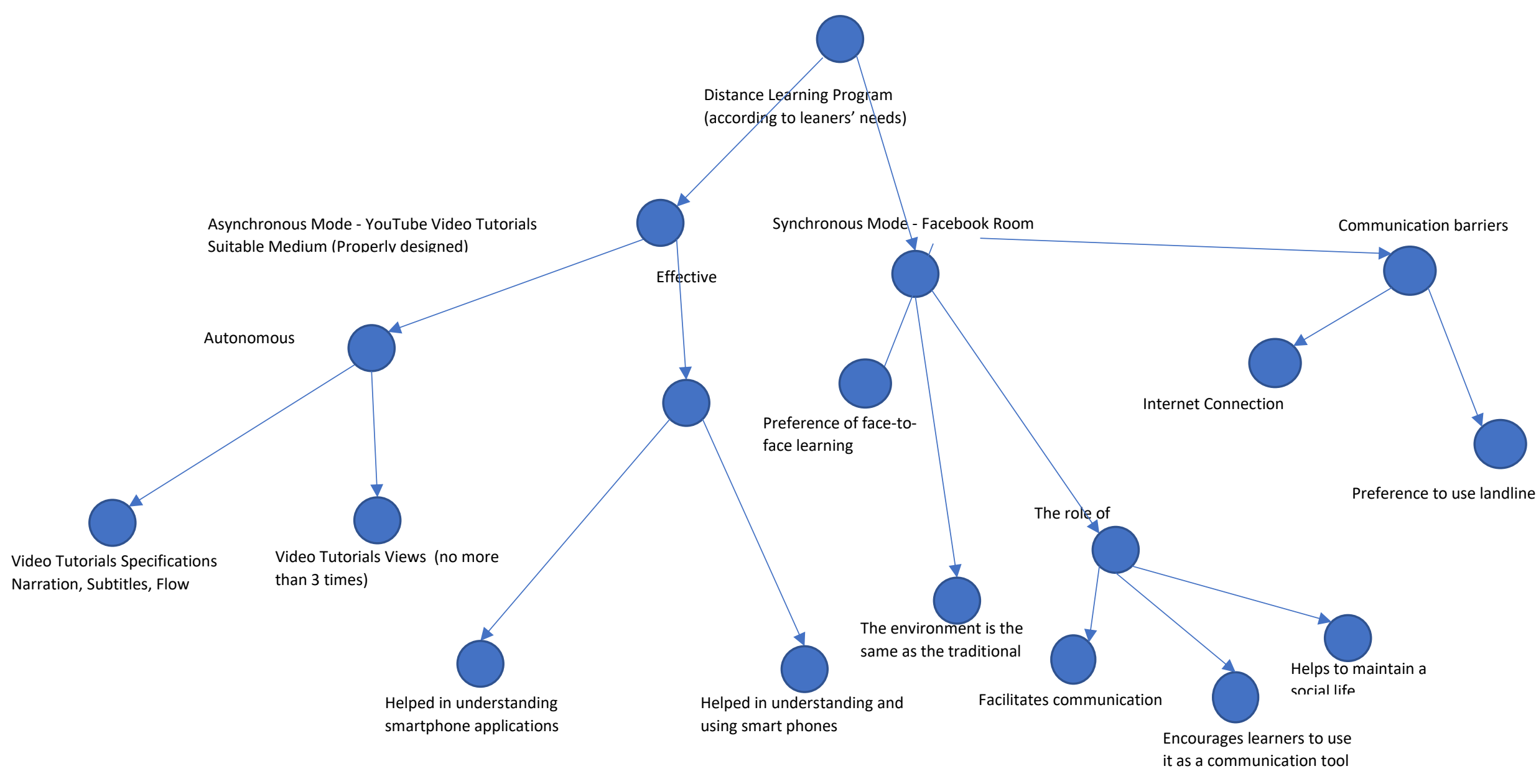

Figure 5: Study's Thematic Analysis Results in whole 


\title{
5. Future Research
}

Future research could further investigate important factors in adult education, such as the standards that educational material for mobile phones should meet, learner training on the safe use of the internet and the use of social media to develop social relationships. Additionally, future research could focus on intergenerational learning, where younger and older learners can learn together and from each other by exploring historical events and customs and practices that are relevant to them.

\section{Conclusion}

This study has shown that mobile phones and social media can be used to educate older people on the use of apps in their daily lives to promote communication and social networking. The appropriate design of an educational program, which should be tailored to the educational needs of the elderly to ensure that the optimal education method and material are adopted, plays a crucial role in the effectiveness of the education process. According to the results of the present study, older women prefer asynchronous learning, as it offers flexibility in terms of time, space and pace, while they also acknowledge the advantages of synchronous learning and highlight the important role of the instructor in facilitating learning and promoting communication between learners. Moreover, the results showed, that designers and educators can plan learning programs by including short video tutorials with narration and subtitles as an effective educational mean and by using Facebook as a learning platform in order to engage learners and promote educatorlearner communication.

\section{Acknowledgements}

We would like to thank all the ladies who participated in this study.

\section{Conflict of Interest Statement}

The authors declare no conflicts of interests.

\begin{abstract}
About the Authors
Dr. Venetis Kanakaris was born in Komotini, Greece. He received the B. Eng. degree in Electronic Engineering from Lamia Technical University, Greece, the B. Eng. degree in Electrical Engineering from Kavala Technical University, Greece, in 2004, and the M.Sc. degree in Telecommunications and Technologies from Technical University of Gabrovo, Bulgaria, in 2006. Also, received his PhD from University of Portsmouth in 2012, U.K. as well as the M.Ed. from Open University of Greece in 2013. Since October of 2016, is adjunct Lecturer at International Hellenic University, Dept. of Computer Science, Kavala, Greece. His current research interests lie in energy ad hoc routing protocols and lowpower underwater wireless sensor protocol architecture OSINT techniques, LORA infrastructures, BlockChain technologies in farming and agriculture. Besides the
\end{abstract}


engineering research interests, he focusses on lifelong learning sector as well as the adult education. At the present he is postdoctoral student at School of Early Childhood Education, Aristotle University of Thessaloniki in Greece.

Maria Pavlis-Korres obtained a university degree in Political Science in Athens. From 1983 until 2018 she worked on Adult Education in the Greek Ministry of Education. Since 2005 her research interests are focused on e-learning and she became a PhD student in the University of Alcalá, Spain. In 2008 she obtained her Advance Studies Degree from the Computer Science Department of the University of Alcalá and in 2010 she obtained her PhD with honors. Since 2012 she is a member of the scientific staff of Hellenic Open University and teaches in "Adult Education" and "Education Science" postgraduate programs. In June 2017 she has been elected Associate Professor in the Pedagogical Sector of the Aristotle University of Thessaloniki to teach "Lifelong Learning and non-Formal Education". She has published several articles, chapters and books on Roma, adult education and e-learning. Her current interests are focused on design, development and evaluation of educational projects for adults, face to face and e-learning, as well as the group dynamics in an online environment and the appropriate use of communication tools in order to promote interaction in an online environment.

\section{References}

Aydin, S. (2012). A review of research on Facebook as an educational environment. Educational Technology research and development, 60(6), 1093-1106.

Boulos, M. N., Wheeler, S., Tavares, C., \& Jones, R. (2011). How smartphones are changing the face of mobile and participatory healthcare: an overview, with example from eCAALYX. Biomedical Engineering Online, 10(1), 24.

Boulton-Lewis, G. M., Buys, L., Lovie-Kitchin, J., Barnett, K., \& David, L. N. (2007). Ageing, learning, and computer technology in Australia. Educational Gerontology, 33(3), 253-270.

Braun, V., \& Clarke, V. (2006). Using thematic analysis in psychology. Qualitative research in psychology, 3(2), 77-101.

Braun, V., \& Clarke, V. (2012). Thematic analysis. In H. Cooper, P. M. Camic, D. L. Long, A. T. Panter, D. Rindskopf, \& K. J. Sher (Eds), APA handbook of research methods in psychology, Vol. 2: Research designs: Quantitative, qualitative, neuropsychological, and biological (pp. 57-71). Washington, DC: American Psychological Association.

Carpenter, B. D., \& Buday, S. (2007). Computer use among older adults in a naturally occurring retirement community. Computers in Human Behavior, 23(6),3012-3024.

Chaffin, A. J., \& Harlow, S. D. (2005). Cognitive learning applied to older adult learners and technology. Educational Gerontology, 31(4), 301-329

Chen, Y., \& Persson, A. (2002). Internet use among young and older adults: relation to psychological well-being. Educational Gerontology, 28(9), 731-744. 
Chen, X., Wang, F., You, Z., Wang, X., Tao, C., \& Liu, J. (2017). Design of interactive tutorials on mobile applications for chinese middle-aged and older adults. Art and Design Review, 5(03), 162-180.

Chugh, R., \& Ruhi, U. (2018). Social media in higher education: A literature review of Facebook. Education and Information Technologies, 23(2), 605-616.

Cohen, L., Manion, L., \& Morrison, K. (2013). Research methods in education (7th ed.). Routledge. doi:10.4324/9780203720967

Cotten, S. R., Ford, G., Ford, S., \& Hale, T. M. (2012). Internet use and depression among older adults. Computers in Human Behavior, 28(2), 496-499.

Cotten, S. R., Ford, G., Ford, S., \& Hale, T. M. (2014). Internet use and depression among retired older adults in the United States: a longitudinal analysis. The Journals of Gerontology Series B: Psychological Sciences and Social Sciences, 69(5), 763-771.

Digmayer, C., \& Jakobs, E. M. (2012, March). Interactive video tutorials as a tool to remove barriers for senior experts in online innovation contests. In Proceedings of INTED2012 Conference (pp. 5-7).

Digmayer, C., \& Jakobs, E. M. (2012). Help features in community-based open innovation contests. Multimodal video tutorials for the elderly. In Proceedings of the 30th ACM international conference on Design of communication, (pp. 79-88).

Dorin, M. (2007). Online education of older adults and its relation to life satisfaction. Educational Gerontology, 33(2), 127-143.

Döş, B. (2017). Using Asynchronous Discussion Groups To Support Teaching In Higher Education. Distance Learning, Simulation and Communication, 41.

Elo, S., \& Kyngäs, H. (2008). The qualitative content analysis process. Journal of advanced nursing, 62(1), 107-115.

Gao, S., Yang, Y., \& Krogstie, J. (2015). The adoption of smartphones among older adults in China. In K. Liu, K. Nakata, W. Li, \& D. Galarreta (Eds.), Information and Knowledge Management in Complex Systems. ICISO 2015. IFIP Advances in Information and Communication Technology (pp. 112-122). Heidelberg, Germany: Springer.

Githens, R. P. (2007). Older adults and e-learning: Opportunities and barriers. Quarterly Review of Distance Education, 8(4), 329-338.

Greenhow, C., Chapman, A. (2020). Social distancing meet social media: digital tools for connecting students, teachers, and citizens in an emergency. Information and Learning Sciences, 121 (5/6), 341-352.

Grindrod, K. A., Gates, A., Dolovich, L., Slavcev, R., Drimmie, R., Aghaei, B., et al. (2014). ClereMed: lessons learned from a pilot study of a mobile screening tool to identify and support adults who have difficulty with medication labels. JMIR mHealth and uHealth, 2(3).

Hung, J.-Y., \& Lu, K.-S. (2014). Research on the healthy lifestyle model, active ageing, and loneliness of senior learners. Educational Gerontology,40(5), 353-362.

Kanakaris, V., \& Pavlis Korres, M. (2020). Investigating the Educational Needs of Elderly People Within the Scope of an Educational Program on the Use of Social Media Networks by Smartphones. In P.O. De Pablos, M.D. Lytras, Z. Xi, (Eds.), IT and the 
Development of Digital Skills and Competences in Education (pp.100-121). IGI Global. https://doi:10.4018/978-1-7998-4972-8.ch007

Klimova, B., Simonova, I., Poulova, P., Truhlarova, Z., \& Kuca, K. (2016). Older people and their attitude to the use of information and communication technologies-a review study with special focus on the Czech Republic (older people and their attitude to ICT). Educational Gerontology, 42(5), 361-369.

Lin, Y.-Y., \& Huang, C.-S. (2013). Policies and practices in educational gerontology in Taiwan. Educational Gerontology, 39(4), 228-240.

Ma, Q., Chan, A. H. S., \& Chen, K. (2016). Personal and other factors affecting acceptance of smartphone technology by older Chinese adults. Applied Ergonomics, 54, 62-71. doi:10.1016/j.apergo.2015.11.015.

Mackowicz, J., \& Wnek-Gozdek, J. (2016). "It's never too late to learn"-how does the Polish U3A change the quality of life for seniors? Educational Gerontology, 42(3), 186197.

Manca, S., \& Delfino, M. (2021). Adapting educational practices in emergency remote education: Continuity and change from a student perspective. British Journal of Educational Technology. (52), 1394-1413

Miller, M. R. (2016). My ipad for seniors. Indianapolis, IN: Que Publishing.

Mulenga, D., \& Liang, J. S. (2008). Motivations for older adults' participation in distance education: A study at the National Open University of Taiwan. International Journal of Lifelong Education, 27(3), 289-314.

National Development Council of Taiwan. (2017). Digital divide in Taiwan 2016. Retrieved from https://www.ndc.gov.tw/ cp.aspx?n=55C8164714DFD9E9

Nowell, L. S., Norris, J. M., White, D. E., \& Moules, N. J. (2017). Thematic analysis: Striving to meet the trustworthiness criteria. International journal of qualitative methods, 16(1)

Pavlis Korres, M., Karalis, Th., Leftheriotou, P., \& García Barriocanal, E. (2009). IntegratingAdults' Characteristics and the Requirements for Their Effective Learning in an E-Learning Environment. In M. Lytras, Best Practices for the Knowledge Society. WSKS (pp.570-584). Springer.

Pavlis-Korres, M., \& Leftheriotou, P. (2016). Building Interaction in Adults' Online Courses: A Case Study on Training E-Educators of Adutls. In Kyei-Blankson, L., Ntuli, E. \& Agyeman, C., Handbook of Research on Strategic Management of Interaction, Presence and Participation in Online Courses (pp. 185-215). USA: IGIGlobal.

Pavlis Korres, M., \& Leftheriotou, P. (2020). Design of Face to Face and E-learning non-Formal Education Programs for Adults. Athens: Ypsilon. (in Greek)

Shapira, N., Barak, A., \& Gal, I. (2007). Promoting older adults' well-being through Internet training and use. Aging $\mathcal{E}$ Mental Health, 11(5), 477-484. https://doi.org/10.1080/13607860601086546.

Stemler, S. E. (2015). Content analysis. Emerging trends in the social and behavioral sciences: An Interdisciplinary, Searchable, and Linkable Resource, 1-14. 
Swift, K. R. (2018). The seven principles for good practice in (asynchronous online) legal education. Mitchell Hamline L. Rev., 44, 105.

Tsai, H.-Y. S., Shillair, R., Cotten, S. R., Winstead, V., \& Yost, E. (2015). Getting grandma online: Are tablets the answer for increasing digital inclusion for older adults in the U.S.? Educational Gerontology, 41(10), 695-709.

Ulla, M. B., \& Perales, W. F. (2021). Facebook as an integrated online learning support application during the COVID19 pandemic: Thai university students' experiences and perspectives. Heliyon, e08317

Ulla, M. B., \& Perales, W. F. (2020). The adoption of Facebook as a virtual class whiteboard: Promoting EFL students' engagement in language tasks. TESOL Journal, 1-4.

Wang, W.-N. (2017). Continued learning in an aging society: A university-community collaborative educational intervention in Taiwan. In C. S. Collins (Ed.), Universitycommunity engagement in the Asia pacific: Public benefits beyond individual degrees (pp. 103-122). New York, NY: Palgrave Macmillan.

White, H., McConnell, E., Clipp, E., Bynum, L., Teague, C., Navas, L., et al. (1999). Surfing the net in later life: a review of the literature and pilot study of computer use and quality of life. Journal of Applied Gerontology, 18(3), 358-378.

White, H., McConnell, E., Clipp, E., Branch, L. G., Sloane, R., Pieper, C., \& Box, T. L. (2002). A randomized controlled trial of the psychosocial impact of providing internet training and access to older adults. Aging $\mathcal{E}$ Mental Health, 6(3), 213-221.

WHO. (2002, April). Active ageing. A policy framework. World Health Organization. Retrieved from https://www.who.int/ageing/publications/active ageing/en/

Zheng, R., Spears, J., Luptak, M., \& Wilby, F. (2015). Understanding older adults' perceptions of internet use: An exploratory factor analysis. Educational Gerontology, 41(7), 504-51

Zhou, J., Rau, P.-L. P., \& Salvendy, G. (2014). Older adults' use of smart phones: an investigation of the factors influencing the acceptance of new functions. Behaviour \& Information Technology, 33(6), 552-560. 
Creative Commons licensing terms

Authors will retain the copyright of their published articles agreeing that a Creative Commons Attribution 4.0 International License (CC BY 4.0) terms will be applied to their work. Under the terms of this license, no permission is required from the author(s) or publisher for members of the community to copy, distribute, transmit or adapt the article content, providing a proper, prominent and unambiguous attribution to the authors in a manner that makes clear that the materials are being reused under permission of a Creative Commons License. Views, opinions and conclusions expressed in this research article are views, opinions and conclusions of the author(s). Open Access Publishing Group and European Journal of Open Education and E-learning Studies shall not be responsible or answerable for any loss, damage or liability caused in relation to/arising out of conflict of interests, copyright violations and inappropriate or inaccurate use of any kind content related or integrated on the research work. All the published works are meeting the Open Access Publishing requirements and can be freely accessed, shared, modified, distributed and used in educational, commercial and non-commercial purposes under a Creative Commons Attribution 4.0 International License (CC BY 4.0). 